\title{
Incidence and antibiotic sensitivity pattern of New Delhi metallo - B - latamase - 1 (NDM-1) producers among carbapenem resistant enterobacteriaceae in a tertiary care teaching hospital, Bareilly: A cross sectional study
}

\author{
Anju Saxena ${ }^{1}$, Sujata Singh², Rahul Kumar Goyal ${ }^{3}$, Jaspreet Kaur ${ }^{4}$, Sumit Saxena ${ }^{5, *}$ \\ ${ }^{1,5}$ Assistant Professor, ${ }^{2}$ Professor \& HOD, ${ }^{3}$ Professor, ${ }^{4}$ Research Associate, ${ }^{1,2,4}$ Dept. of Pharmacology, ${ }^{3}$ Dept. of \\ Microbiology, ${ }^{5}$ Dept. of Community Medicine,
}

*Corresponding Author:

Email: drsumitsaxena22@gmail.com

\begin{abstract}
Objectives: Antibiotic resistance in microorganisms has become a critical health issue these days and has evolved to become a worldwide health threat. Carbapenem-resistant Enterbactriaceae (CRE) is one example which really is a nightmare bacteria, resistant to most, and in some cases all, antibiotics. The commonest cause is ellaboration of various types of carbapenemases amongst which the recent detection of New Delhi metallo beta-lactamase-1 (NDM-1), a superbug, further compounded the problem. So the present study was undertaken to study the pattern of carbapenem resistance and role of carbapenamase (blaNDM-1) gene towards it.

Materials and Methods: The study was a cross sectional study conducted in the department of Pharmacology in collaboration with Microbiology and Biochemistry, SRMS IMS, Bareilly. All clinical isolates, their sensitivity pattern and MBL detection of carbapenem resistant ESBLS were obtained from Microbiology records. blaNDM-1 gene was detected by PCR.

Results: A total of 312 ESBL producers were isolated. Out of which 42 were found to be carbapenem resistant. Amongst these $19.05 \%$ (8) were found to be MBL positive and $5(62.5 \%)$ MBL positive isolates were NDM positive. ESBL producers were sensitive to very few antibiotics and NDM producers were sensitive only to polypeptide antibiotics.

Conclusion: ESBL is a great problem for gram negative organisms and now the frequency of carbapenem resistance is high which has limited antibiotics available for the treatment. Furthermore carbapenemase (blaNDM-1) gene has also shown a substantial role. This high incidence of antibacterial resistance demands development of newer antibiotics.
\end{abstract}

Keywords: Carbapenem resistance, ESBL, blaNDM-1, MBL.

\section{Introduction}

Resistance to $\beta$-lactams is a long recognised problem in Gram-negative bacteria ${ }^{1}$ and with the introduction of new classes of $\beta$-lactams, novel $\beta$ lactamases have emerged. ${ }^{1,2}$ Carbapenem resistance has become a growing problem over the last decade with the emergence of readily transferable plasmid mediated carbapenem-hydrolysing $\beta$-lactamases and has posed a threat to the antimicrobial world. ${ }^{3,4}$ According to a WHO report the "... world is heading towards a postantibiotic era in which many common infections will no longer have a cure and once again kill unabated". Carbapenems are the agents of last resort against many multi drug resistant, gram negative bacteria. Pseudomonas and Acinetobacter baumannii have significant carbapenem resistance. These enzymes confer resistance to the other beta-lactam agents as well, including extended spectrum cephalosporins. Metallo beta lactamases (MBLs) are one such type of carbapenemase, that are characterized by the ability to hydrolyze carbapenems. A novel MBL named NDM-1 (New Delhi metallo- $\beta$-lactamase) was identified from Klebsiella pneumoniae (strain 05-506) and Escherichia coli isolates recovered from a Swedish patient transferred from India. ${ }^{6}$ Since then, there have been an increasing number of infections in patients from India, Pakistan and the United Kingdom. ${ }^{7}$ Various studies conducted in the past few years have shown its dissemination across the gram negative organisms and included E.coli, Klebsiella pneumonia, Citrobacter. ${ }^{8}$ Effective surveillance systems have been put in place in some countries to track the emergence and spread of resistance to anti-infective. Such surveillance has been able to bring about changes in national policies and practices. With this background, the present study was undertaken to study the variability in antimicrobial resistance pattern in carbapenem resistant organisms amongst indoor and outdoor patients and genotypic identification of carbapenamase (bla $\mathrm{NDM}_{\mathrm{N}-1}$ ) gene.

\section{Materials and Methods}

Study design: Cross sectional study.

Place of study: Department of Pharmacology in collaboration with Department of Microbiology and Department of Biochemistry, Shri Ram Murti Smarak Institute of Medical Sciences, Bhojipura, Bareilly.

Study period: April 2013 to March 2014.

Sample: Clinical isolates of various gram positive and gram negative organisms, from sputum, endotracheal tip, tracheal aspirate, urine, pus swab, pus aspirate, bronchial wash, catheter tip, blood, pleural fluid, peritoneal fluid, pericardial fluid, ascitic fluid, sample from shunt tube, corneal swab, intracervical swab, wound tissue, CSF and drainage tip from the inpatients and outpatients of Obstetrics and Gynaecology, Surgery, Medicine, Orthopaedics, Ophthalmology, 
Paediatrics, Casualty, ENT, ICU wards were taken from Microbiology records.

The study was conducted in compliance with the protocol and the Institutional Ethics Research Committee (IERC).

Antimicrobial susceptibility test was done by Kirby-Bauer sensitivity testing method. Carbapenem resistance was analysed amongst various ESBL positive isolates and was grouped into IPD and OPD isolates. The sensitivity of ESBL producing carbapenem resistant organisms was also evaluated and then this was compared between the two groups. Metallo $\beta$ lactamase (MBL) was tested using double disk diffusion test (DDDT) in carbapenem resistant ESBLs. bla NDM-1 gene was detected by PCR for which the positive control was obtained from department of Microbiology, Institute of Medical Sciences, Banaras Hindu University, Varanasi. The primers for this gene were obtained from HiMedia Laboratories Pvt Limited, Mumbai. Samples of carbapenem resistant ESBLs were processed further for PCR. Further we analysed positive

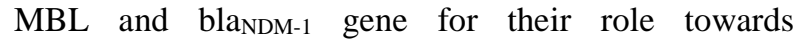
carbapenem resistance and their ultimate contribution towards antibacterial resistance and the available treatment options. The data was analysed using IBM SPSS version 20. Chi-square test was applied wherever applicable to check the significant difference among the different groups. $p$ value of $\leq 0.05$ was considered to be significant.

\section{Results}

Present cross sectional study includes a total of 2008 samples from various clinical departments. Of these 2008 samples, $655(32.62 \%)$ specimen gave significant growth of bacteria while rest were either non-pathogenic or sterile (Fig. 1).

Of the total number of positive isolates 576 $(87.94 \%)$ were from IPD and $79(12.06 \%)$ specimen were from OPD. Majority of the specimen were infected by gram negative bacteria, E.coli (25.3\%) (Fig. 2 ). Out of total positive samples, $47.63 \%$ (312) isolates were ESBL producers (Fig. 3) and out of these, 42 ESBL producing isolates were found to be carbapenem resistant and maximum incidence was observed with Acinetobacter followed by Pseudomonas and none of the Proteus isolates were carbapenem resistant. Carbapenem resistance was significantly more in IPD isolates as compared to OPD isolates $(\mathrm{p}<0.05)$ (Table 1). On further analysing the sensitivity pattern of carbapenem resistant isolates, it was found that E.coli was sensitive in decreasing order to $\mathrm{Cl} \& \mathrm{~PB}$ followed by Cot \& C while Klebsiella \& Pseudomonas were sensitive only to $\mathrm{Cl} \& \mathrm{~PB}$. (Table $2 \mathrm{a} \& 2 \mathrm{~b}$ ). Out of 42 carbapenem resistant ESBL positive isolates, 8 were MBL positive \& when genotypic identification (Fig. 46) was done for carbapenemase gene it was found that 5 of MBL positive isolates were NDM positive (Table 3).
The data also reveals that there is a positive correlation between carbapenem resistant ESBL \& MBL and also between MBL \& NDM ( $\mathrm{p}<0.05)$.

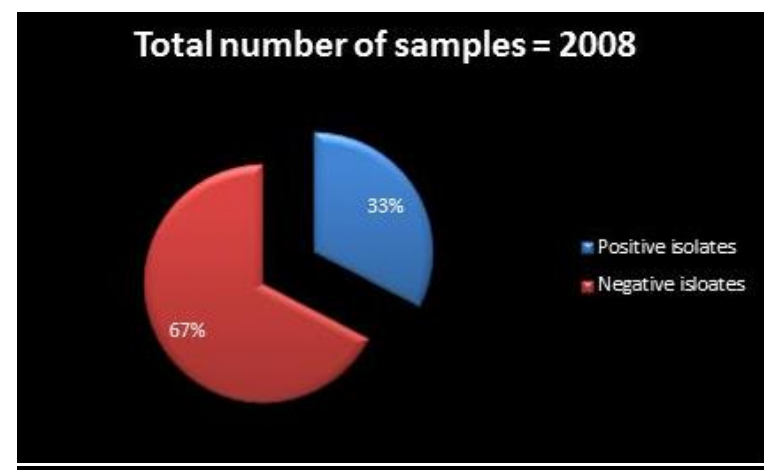

Fig. 1: Total number of samples

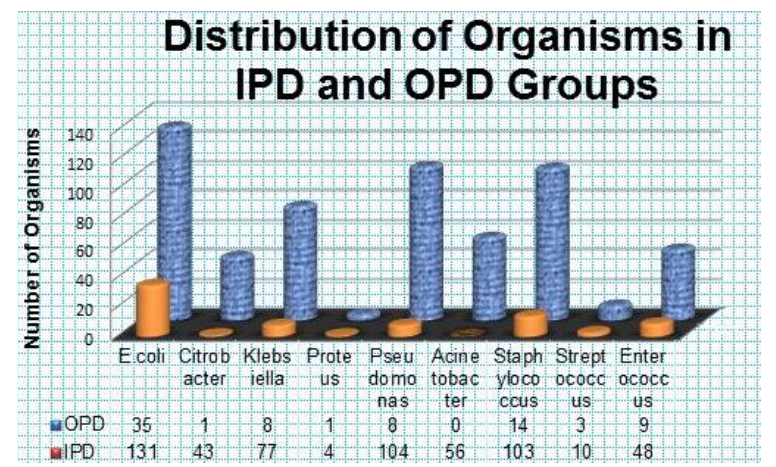

Fig. 2: Distribution of organisms in IPD and OPD groups

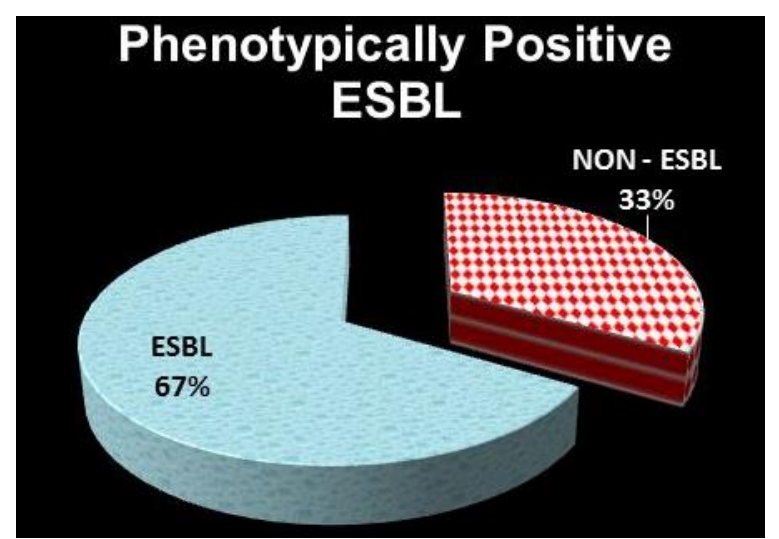

Fig. 3: Phenotypically positive ESBL

Table 1: Distribution of Carbapenem Resistant ESBL Organisms

\begin{tabular}{|l|c|c|c|c|c|}
\hline \multirow{2}{*}{ Organism } & \multicolumn{2}{|c|}{ IPD } & \multicolumn{2}{c|}{ OPD } & $\begin{array}{c}\text { p- } \\
\text { value }\end{array}$ \\
\cline { 2 - 6 } & No. & $\%$ & No. & $\%$ & \\
\hline Acinetobacter & 12 & 27.91 & 0 & 0 & N.A. \\
\hline Citrobacter & 7 & 21.21 & 0 & 0 & N.A. \\
\hline Proteus & 0 & 0 & 0 & 0 & N.A. \\
\hline Klebsiella & 8 & 13.56 & 0 & 0 & $>0.05$ \\
\hline Pseudomonas & 9 & 25 & 0 & 0 & $>0.05$ \\
\hline E.coli & 4 & 3.54 & 2 & 10.53 & $>0.05$ \\
\hline
\end{tabular}


Table 2a: Antibiotic Sensitivity Pattern of Carbapenem Resistant ESBL Positive Enterobacteriaceae

\begin{tabular}{|c|c|c|c|c|c|c|c|c|c|}
\hline & \multicolumn{2}{|c|}{ (2) } & \multirow[t]{2}{*}{ p-value } & \multicolumn{2}{|c|}{ Lebsiella } & \multirow[t]{2}{*}{ p-value } & \multicolumn{2}{|c|}{ (2) } & \multirow[t]{2}{*}{ p-value } \\
\hline & $\begin{array}{c}\text { IPD } \\
(n=4)\end{array}$ & $\begin{array}{c}\text { OPD } \\
(n=2)\end{array}$ & & $\begin{array}{c}\text { IPD } \\
(n=8)\end{array}$ & $\begin{array}{c}\text { OPD } \\
(\mathbf{n}=\mathbf{0})\end{array}$ & & $\begin{array}{c}\text { IPD } \\
(\mathbf{n}=7)\end{array}$ & $\begin{array}{c}\text { OPD } \\
(\mathbf{n}=0)\end{array}$ & \\
\hline Cip & 0 & 0 & NA & 0 & 0 & NA & 0 & 0 & NA \\
\hline Le & 0 & 0 & NA & 1 & 0 & NA & 1 & 0 & NA \\
\hline Of & 0 & 0 & NA & 0 & 0 & NA & 0 & 0 & NA \\
\hline AK & 0 & 0 & NA & 0 & 0 & NA & 0 & 0 & NA \\
\hline Gen & 0 & 0 & NA & 0 & 0 & NA & 0 & 0 & NA \\
\hline $\mathbf{T b}$ & 0 & 0 & NA & 0 & 0 & NA & 0 & 0 & NA \\
\hline Tet & 0 & 1 & $>0.05$ & 1 & 0 & NA & 1 & 0 & NA \\
\hline C & 1 & 0 & NA & 0 & 0 & NA & 0 & 0 & NA \\
\hline Cot & 1 & 1 & $>0.05$ & 0 & 0 & NA & 0 & 0 & NA \\
\hline CFS & 0 & 0 & NA & 0 & 0 & NA & 0 & 0 & NA \\
\hline AMC & 0 & 0 & NA & 0 & 0 & NA & 0 & 0 & NA \\
\hline Pit & 0 & 0 & NA & 0 & 0 & NA & 0 & 0 & NA \\
\hline Nit & - & - & - & 0 & 0 & NA & 0 & 0 & NA \\
\hline $\mathrm{Cl}$ & 4 & 2 & $>0.05$ & 7 & 0 & NA & 6 & 0 & NA \\
\hline PB & 4 & 2 & $>0.05$ & 7 & 0 & NA & 6 & 0 & NA \\
\hline
\end{tabular}

Table 2b: Antibiotic Sensitivity Pattern of Carbapenem Resistant ESBL Positive Enterobacteriaceae

\begin{tabular}{|c|c|c|c|c|c|c|}
\hline & \multicolumn{2}{|c|}{$\mathbf{p}$-value } & \multicolumn{2}{|c|}{ p-value } \\
\cline { 2 - 3 } & $\begin{array}{c}\text { IPD } \\
(\mathbf{n = 1 2})\end{array}$ & $\begin{array}{c}\text { OPD } \\
(\mathbf{n = 0})\end{array}$ & & $\begin{array}{c}\text { IPD } \\
(\mathbf{n = 9})\end{array}$ & $\begin{array}{c}\text { OPD } \\
(\mathbf{n = 0})\end{array}$ & \\
\hline Cip & 0 & 0 & NA & 0 & 0 & NA \\
\hline Le & 0 & 0 & NA & 0 & 0 & NA \\
\hline Of & 0 & 0 & NA & 0 & 0 & NA \\
\hline AK & 0 & 0 & NA & 1 & 0 & NA \\
\hline Gen & 0 & 0 & NA & 1 & 0 & NA \\
\hline Tb & 0 & 0 & NA & 1 & 0 & NA \\
\hline Tet & 0 & 0 & NA & - & - & - \\
\hline C & 0 & 0 & NA & - & - & - \\
\hline Cot & 0 & 0 & NA & - & - & - \\
\hline CFS & 1 & 0 & NA & 0 & 0 & NA \\
\hline AMC & 0 & 0 & NA & 0 & 0 & NA \\
\hline Pit & 0 & 0 & NA & 1 & 0 & NA \\
\hline Nit & 0 & 0 & NA & - & - & - \\
\hline Cl & 12 & 0 & NA & 9 & 0 & NA \\
\hline PB & 12 & 0 & NA & 9 & 0 & NA \\
\hline
\end{tabular}

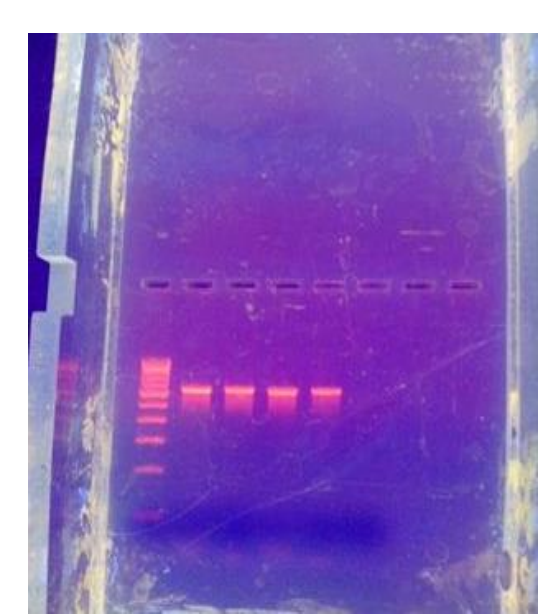

Fig. 4: Positive control of NDM -1

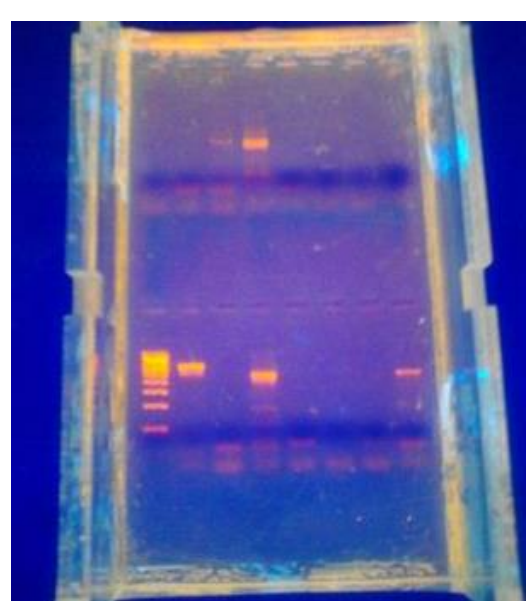

Fig. 5: Four NDM-1 positive strains 


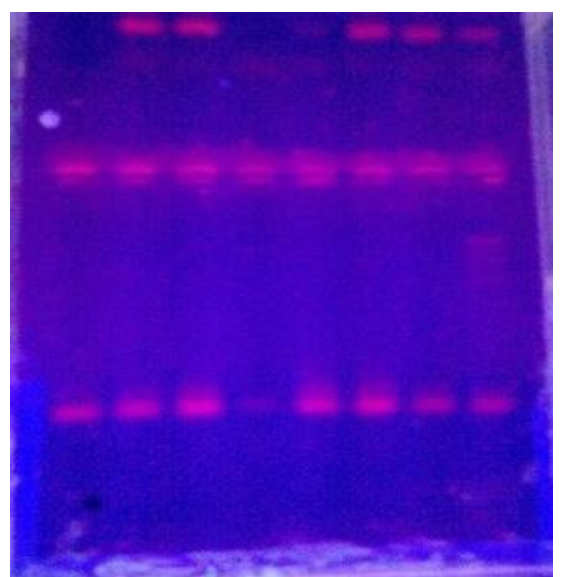

Fig. 6: One NDM-1 positive strain

Table 3: Correlation of MBL and NDM

\begin{tabular}{|l|c|c|}
\hline & $\begin{array}{c}\text { NDM } \\
\text { positive }\end{array}$ & $\begin{array}{c}\text { NDM } \\
\text { negative }\end{array}$ \\
\hline MBL positive (8) & 5 & 3 \\
\hline MBL negative (39) & 0 & 39 \\
\hline
\end{tabular}

p - value $<0.05$

\section{Discussion}

Knowledge on local antibacterial resistance trends among various isolates is important not only in guiding clinicians to prescribe appropriate antibiotics but also for evidence based recommendations in empirical antibiotic treatment. The current study described the role of ESBL contributing towards antibacterial resistance and the incidence of carbapenem resistance due to bla $\mathrm{NDM}_{1}$ gene.

In India, the prevalence of ESBLs has been reported since the 1990s. ${ }^{9-12}$ The phenotypic data generated in the current study indicates a high prevalence $(47.63 \%)$ of ESBL producers. This number is less than that previously reported by Dalela et al in 2012 and Narayanswamy and Mallika in 2011. ${ }^{12,13}$ Out of the total ESBL producers, maximum frequency was observed with Escherichia coli $(79.52 \%)$ in the current study which is in accordance to a study conducted in Chennai which showed ESBL production among 75.5\% Escherichia coli isolates ${ }^{14}$ while $49.32 \%$ Escherichia coli were ESBL producer in a study conducted in Mumbai by Aruna et al 2012.

The reports presented by different authors clearly indicate that the prevalence of ESBL producing organisms among clinical isolates vary greatly geographically and rapidly changing over time..$^{15,16}$

Antibiotic options in the treatment of ESBLproducing organisms are extremely limited. Carbapenems are the treatment of choice for serious infections due to ESBL-producing organisms. ${ }^{17}$ and are often used as "last line agents" or "antibiotics of last resort".

Our study also analysed that 42 ESBL positive organisms are carbapenem resistant leaving only polypeptide antibiotics and a trickle of drugs for their treatment. Our results were found to be similar to study conducted in 2013 in Mumbai which showed 12.26\% Enterobacteriaceae to be carbapenem resistant. ${ }^{18}$ Other studies conducted in various parts of India showed carbapenem resistance in Enterobacteriaceae to be in the range of $7.87 \%$ to $51 \% .{ }^{19-21}$ This variability could be due to difference in study design, study population, geographical distribution and different drug pressure in community.

When the sensitivity pattern of these carbapenem resistant ESBL positive isolates was analysed it was noted that $87.5 \%$ Klebsiella was sensitive only to polypeptide antibiotics and no drugs were available for the rest $12.5 \%$ making them eternal and thereby spreading resistance to other organisms by genetic transfer. In the same way, carbapenem resistant ESBL producing Acinetobacter and Pseudomonas were found to be sensitive only to polypeptide antibiotics suggesting need for development of innovative and improved drugs and their vigilant use.

As carbapenems have long been considered the antibiotic class of last resort in the treatment of infections caused by multidrug-resistant organisms, the dissemination of carbapenem resistance among pathogenic bacteria has been declared a "global sentinel event". ${ }^{22}$

The current condition suggests judicious and rational use of the antibiotics so that the resistance rate is minimised. Many countries have also given Standard treatment guidelines (STG) to direct choice of antibiotics.

Carbapenem resistance due to acquired carbapenemases has emerged and spread worldwide since the early $2000 \mathrm{~s}^{1}$ and the number of bacteria that produce metallo- $\beta$-lactamase (MBL) is on the rise. ${ }^{2}$ In the present study, $19.05 \%$ carbapenem resistant ESBL positive isolates were MBL positive.

In the present study we also observed that out of 8 MBL positive strains, $62.5 \%$ isolates harbaoured blanDM-1 gene. Similar to this study, Seema et al., reported $54 \quad(84.38 \%) \quad \mathrm{NDM}-1$ producing Enterobacteriaceae. ${ }^{23}$ Out of this, $33.33 \%$ were found in E.coli and $37.5 \%$ were found in Klebsiella which was comparable to a study conducted by Deshpande et al which reported the incidence of NDM - 1 in 9 E.coli isolates among 24 carbapenem resistant Enterobacteriaceae $(37.5 \%)$ in a tertiary care centre in Mumbai. ${ }^{24}$ Another northeastern study by Bora et al, $2013^{25}$ showed $5.2 \%$ bla $_{\text {NDM-1 }}$ gene in E.coli and a study carried out in the intensive care unit (ICU) and wards of Sir Ganga Ram Hospital, Delhi showed $8.1 \%$ overall prevalence of NDM - 1 in E.coli isolates. ${ }^{26}$ Plasmid transfer is one of the major factors in disseminating antibacterial drug-resistant bacteria. This difference in the percentage of presence of bla $\mathrm{NDM}_{\mathrm{N}-1}$ gene could be because of the different geographical areas. ${ }^{27}$ These reports indicate an alarming risk of rapid dissemination of NDM-1. Identification of a significant number of 
NDM - 1 producer in E.coli is an additional source of concern as it suggests that the resistance is being disseminated in the environment as well as in the hospital. E.coli is the cause of many community acquired infections, such as diarrhoea and urinary tract infections. Overcrowding coupled with poor hygiene, difficulty in obtaining potable water, poor sanitation, sale of non - prescribed antibiotics (self-medication) and weak hospital antibiotic policies may be contributing factors for selecting and facilitating the spread of NDM -1 producers. The possible spread of NDM - 1 producer reinforces the urgent need to develop novel anti - gram - negative molecules and the implementation of a worldwide network of sentinel laboratories for antibiotic resistance surveillance. The need of the hour is to preserve antibiotics and trials should be encouraged to study the impact of carbapenem restriction for treatment of ESBLs on the incidence of $\mathrm{MBL}$ - producing gram negative pathogens.

Careful surveillance of antibiotic use and resistance patterns could aid in the development of antimicrobial guidelines to improve appropriate use and delay the spread of resistance and thus surveillance studies should be done periodically and in different geographical areas and the results should be made available to all the treating physicians in order to guide proper use of antibiotics.

\section{Conclusion}

Antibiotic resistance is a serious public health threat that is on the rise - bacteria that are resistant to our best antibiotics continue to emerge. ESBL and now bla NDM-1 (carbapenemase) gene has also found its place in the world of antibiotic resistance.This high incidence of antibacterial resistance demands development of newer antibiotics.

\section{Acknowledgement}

The authors would like to acknowledge Shri Ram Murti Smarak Institute of Medical Sciences, Bareilly for their financial support.

\section{Limitations of the study}

This study was conducted in a limited area and thus may not represent the whole population.

\section{Conflicts of Interest}

There are no conflicts of interest.

\section{References}

1. Rolain MJ, Panola P, Cornaglia G. New Delhi metallobeta-lactamase (NDM-1): towards a new pandemia?. Clin Microbiol Infect 2010;16:12.

2. Schlesinger SR, Lahousse MJ, Foster OT, Kun Kim S. Metallo- $\beta$-Lactamase and Aptamer-Based Inhibition. Pharmaceuticals 2011;4:419-28.
3. Nordmann P, Poirel L. Emerging carbapenemases in Gram-negative aerobes. Clinical Microbiology and Infection. 2002;8:321-331.

4. Kyle D. Brizendine, Sandra S. Richter, Eric D. Cober, David van Duinc. Carbapenem-Resistant Klebsiella pneumoniae Urinary Tract Infection following Solid Organ Transplantation. Antimicrobial Agents and Chemotherapy. 2015;59(1):553-7.

5. G-Science Academies Statements 2013. Drug resistance in Infectious Agents - a Global threat to Humanity. [online]. 2011 [cited 2014 Aug 26]; Available from:

6. URL www.who.int/pmnch/media/membernews/2011/2011040 7_who_whd/en/

7. Yong, D., M. A. Toleman, C. G. Giske, H. S. Cho, K. Sundman, K. Lee, and T. R. Walsh. Characterization of a new metallo-_-lactamase gene, blaNDM-1, and a novel erythromycin esterase gene carried on a unique genetic structure in Klebsiella pneumoniae sequence type 14 from India. Antimicrob. Agents Chemother.2009;53:5046-54.

8. Zarfel G, Hoenigl M, Leitner E, Slazer HJF, Feierl G, Marsoud L. Emergence of New Delhi Metallo- $\beta$ Lactamase, Austria. Emerg Infect Dis 2011;17(1):129-30.

9. Walsh TR, Toleman MA, Poirel L, Nordmann P. Metallo$\beta$-lactamases: the quiet before the storm? Clin Microbiol Rev 2005;18:306-25.

10. Revathi G, Singh S. Detection of expanded spectrum cephalosporin resistance due to inducible lactamases in hospital isolates. Indian J Med Microbiol 1997;15:113-5.

11. Karim A, Poirel S, Nagarajan, Nordmann P. Plasmid mediated extended-spectrum beta-lactamase from India and gene association with insertion sequence ISEcp1. FEMS Microbiol Lett 2001;201:237-41.

12. Aggarwal R, Chaudhary U, Sikka R. Detection of extended spectrum beta-lactamase production among uropathogens. J Lab Physicians 2009;1:7-10.

13. Narayanaswamy A, Mallika M. Prevalence of extended spectrum beta lactamases in urinary isolates of Escherichia coli in a tertiary care hospital, Chennai, South India. Intl J Med 2011;6:39-43.

14. Dalela G, Gupta S, Jain DK, Mehta P. Antibiotic resistance pattern in uropathogens at a tertiary care hospital at Jhalawar with special reference to ESBL, AmpC $\beta$ - lactamases and MRSA production. J Clin Diagn Res 2012;6:645-51.

15. Maya AS, Prabhakar K, Hanna LE, Sarayu YL. Phenotypic and genotypic characterization of extendedspectrum beta-lactamase producing Escherichia coli clinical isolates from Semi urban Area. J Pharm Res 2011;4:6-10.

16. Krishnakumar S, Rajan RA, Babu MM, Bai VD. Antimicrobial susceptibility pattern of extended spectrum of beta lactamase (ESBL) producing uropathogens from pregnant women. India J Med Healthcare 2012;1:188-92.

17. Madhavan A, Jayalakshmi V. Occurrence of extendedspectrum beta-lactamase, AmpC and MBLase producers among multidrug-resistant Enterobacteriaceae causing urinary tract infection in a tertiary health-care teaching hospital. J Acad Clin Microbiologists 2016;18(2):80-5.

18. Paterson DL. Recommendation for treatment of severe infections caused byEnterobacteriaceae producing extended-spectrum $\beta$-lactamases (ESBLs). Clin Microbiol Infect 2000;6:460-3.

19. Nair PK, Vaz MS. Prevalence of carbapenem resistant Enterobateriaceae from a tertiary care hospital in Mumbai, India. J Microbiol Infect Dis 2013;3(4):207-10. 
20. Datta P, Gupta V, Garg S, Chander J. Phenotypic method for differentiation of carbapenemases in

Enterobacteriaceae: study from north India. Indian J Pathol Microbiol 2012;55:357-60.

21. Saini M, Mishra A, Gupta S. Prevalence of Carbapenem Resistance in Gram Negative Bacilli Isolates and Their Antimicrobial Susceptibility Pattern. Int J Med Res Prof 2016; 2(3);28-32.

22. Gupta N, Limbago BM, Patel JB, Kallen AJ. Carbapenem resistant Enterobacteriaceae: epidemiology and prevention. Healthcare Epidemiol 2011;53:60-7.

23. Richet HM, Mohammed J, McDonald LC, Jarvis WR. Building communication networks: International network for the study and prevention of emerging antimicrobial resistance. Emerg Infect Dis 2001;7(2):319-22.

24. Kumari S, Sen MR, Upadhyay S, Bhattacharjee A. Dissemination of the New Delhi metallo-b-lactamase-1 (NDM-1) among Enterobacteriaceae in a tertiary referral hospital in north India. J Antimicrob Chemother 2011;66(5):1-2.

25. Deshpande P, Rodrigues C, Shetty A, Kapadia F, Hedge A, Soman R. New Delhi Metallo-beta lactamase (NDM-1) in Enterobacteriaceae: Treatment options with carbapenems compromised. J Assoc Physicians India 2010;58:147-9.

26. Bora A, Ahmed GU, Hazarika NK, Prasad KN, Shukla SK, Randhawa V, et al. Incidence of blaNDM-1 gene in Escherichia coli isolates at a tertiary care referral hospital in Northeast India. Indian J Med Microbiol 2013;31(3):250-6.

27. Wattal C, Sharma A, Oberoi JK, Datta S, Prasad KJ, Raveendar R. ESBL- An emerging threat to antimicrobial therapy. Microbiology Newsletter Sir Ganga Ram Hospital 2005;10(1):1-8.

28. Rahman M, Shukla SK, Prasad KN, Ovejero CM, Pati BK, Tripathi A et al. Prevalence and molecular characterisation of New Delhi metallo- $\beta$-lactamases NDM-1, NDM-5, NDM-6 and NDM-7 in multidrugresistant Enterobacteriaceae from India. Int J Antimicrob Agents $2014 \mathrm{Jul} ; 44(1): 30-7$. 